\section{Does my dog need a caesarean?}

\section{Angelika Von Heimendahl}

A successful birth, whether vaginal or by Caesarean section, is one of the most important events for a breeder and can define their relationship with their veterinary surgeon. Given the price of some puppies, Caesarean sections are much in demand and it can be difficult for a clinician to defend trying a vaginal birth on ethical grounds. The knowledge of normal physiological parameters around birth are an important tool for a successful outcome.

Due dates and, in cases where progesterone testing for ovulation and mating is not used, a range of possible dates must be discussed. Clients have to understand that canine pregnancy is very short and that even a few days can make a big difference for the survival rates of the puppies.

Breeders may have to be educated in preparing for birth, measuring body temperature and following good practice during parturition. Any information passed on before the birth, such as timings and out-of-hour arrangements, will mean less stress and fewer phone calls when the time comes. A well planned birth goes a long way to avoiding Caesareans!

Normal parameters of the birthing process will be discussed, such as:

\section{- First-stage labour \\ - Second-stage labour \\ - Passage of placentas \\ - Primary and secondary inertia.}

Different pharmacological options can help with induction and during parturition, but also have to be weighed up against the possibility of performing a Caesarean section. Despite the surgical risk for the bitch and puppies, Caesarean sections are highly succesful interventions and offer an immediate solution to many problems that occur during birth

The lecture will give a short overview of the distribution of Caesareans within breeds and how, through good breeding and selection of females, Caesareans can be avoided in the future.

\section{KEY LEARNING OBJECTIVES}

- Understand the decision-making process for

Caesarean sections in the bitch

- Know what the other options are

- Understand about selection of breeding animals

\section{MULTIPLE CHOICE QUESTIONS}

1. A bitch presents with milk in her mammary glands and the breeders reports she has been restless for 2 days. Mating occurred 61, 60 and 59 days ago. The bitch is clearly in whelp with quite a few puppies. What is the best advice to the breeder and what should you do next?

(A) Milk is sign of prolactin secretion which means the pregnancy has come to its physiological end

(B) The time of mating until the day the bitch is presented means that she is within the normal range of gestation and needs to give birth in the next 24 hours

(C) Sperm can survive for several days in the female reproductive tract and lactation is not an indicator for impending parturition

(D) Low doses of oxytocin will help to start the birth and bring on contractions

2. A Bulldog breeder rings up to book an elective Caesarean for a maiden bitch. She has been mated three times over 4 days and no blood progesterone levels were analysed. $\mathrm{He}$ is confident that she took to the first mating and she is obviously pregnant. He would like the section 63 days after the first mating. What are the parameters for an elective Caesarean?

(A) Due to oocyte and sperm survival, mating dates are not reliable to determine the end of pregnancy

(B) 63 days is enough time to produce mature puppies even if she has taken to the second or third mating

(C) Bulldogs cannot give birth naturally so it is better to be realistic and book the Caesarean

(D) $\mathrm{He}$ is an experienced breeder and there have not been problems in the past

3. A bitch had blood progesterone tested and ovulated 64 days ago. She was mated 62 days ago and scanned to be pregnant with at least eight puppies. She shows no sign of impending birth. What are the options?

(A) Due to long sperm survival parturition may still happen in the next few days and the breeder should provide calm support and wait a little longer

(B) Due to the relatively large number of puppies gestation length is longer

(C) Mating occurred at the time oocytes were ready for fertilization and gestation at this point is 62 days, which means the bitch should show signs of parturition

(D) Ovulation and fertilization can occur over several days and this means that gestation length may be longer 